Amyloidosis database containing baseline demographic and clinical data. Data was analysed using a Chi-squared test (GraphPad Prism 7.0).

Results: Between 1990-2000 there were 188 new cases of AA Amyloidosis of which $51(27.1 \%)$ had an underlying diagnosis of rheumatoid arthritis (RA), median age at diagnosis of amyloid was 60 years. $37(19.7 \%)$ had a diagnosis of Juvenile Idiopathic Arthritis (JIA), median age 29 years. Between 2007 and 2017 there were 270 new diagnoses of AA Amyloidosis, of whom 57 had RA (21.1\%), median age 66 years, and $5(1.9 \%)$ had JIA, median age 37 years. Since the widespread use of combination disease-modifying and biologic therapy there has been a significant reduction in the incidence of JIA-associated AA Amyloidosis $(p<0.0001)$. In contrast, the incidence of RA-associated AA Amyloidosis is unchanged $(\mathrm{p}<0.1357)$

Conclusions: Advances in the treatment of JIA have significantly reduced the incidence of AA Amyloidosis in this cohort. In contrast, despite largely the same medication and similar treatment targets, RA continues to be responsible for a substantial proportion of new cases of AA Amyloidosis. One possible explanation for this may be that current assessment of disease activity in RA is sufficient to identify those at risk of joint damage or radiological progression of disease but insufficient for identifying peristent subclinical biochemical inflammation. One approach for reducing this risk may be the routine measure of the AA Amyloid precursor protein, serum Amyloid A protein (SAA) in those with RA, as it is well established that levels of SAA of below $10 \mathrm{mg} / \mathrm{L}$ prevents the accumulation of $A A$ Amyloid in those with inflammatory disease.

[1] Lachmann, H. J., Goodman HJ, Gilbertson JA, Gallimore JR, Sabin CA, Gillmore JD, Hawkins PN (2007). Natural history and outcome in systemic AA amyloidosis. N Engl J Med 356(23): 2361-2371.

Disclosure of Interest: None declared

DOI: 10.1136/annrheumdis-2017-eular.5005

\section{THU0077 ANTI-COLLAGEN TYPE II ANTIBODIES ARE ASSOCIATED WITH AN ACUTE ONSET RHEUMATOID ARTHRITIS PHENOTYPE AND PROGNOSTICATE LOWER DEGREE OF INFLAMMATION}

V.A. Manivel ${ }^{1}$, M. Mullazehi ${ }^{1}$, L. Padyukov ${ }^{2}$, H. Westerlind $^{3}$, L. Klareskog ${ }^{2}$, L. Alfredsson ${ }^{3}, \mathrm{~S}$. Saevarsdottir ${ }^{2}$, J. Rönnelid ${ }^{1} .{ }^{1}$ Immunology genetics and Pathology, Uppsala University, Uppsala; ${ }^{2}$ Rheumatology Unit, Department of Medicine, Karolinska University Hospital and Karolinska Institutet; ${ }^{3}$ Institute of Environmental Medicine, Karolinska Institutet, Stockholm, Sweden

Background: Anti-collagen II antibody (anti-CII) positive RA patients present with early but not persistent signs of inflammation and joint erosions. This early antiCll-dependent phenotype coincides with high anti-Cll levels around the time of RA diagnosis, whereafter anti-CII levels drop. Our previous studies showed that this phenotype is associated with in vitro cytokine production by monocytes, activation of granulocytes, and enhanced chemokine production by monocyte/granulocyte cocultures, stimulated with anti-CII containing immune complexes. These in vitro findings argue that elevated anti-CIl levels at time of RA diagnosis are functionally related to the corresponding acute onset RA phenotype.

Objectives: Our previous comparison done in a small RA cohort $(n=274)$ describe that anti-cyclic citrullinated peptide 2 (anti-CCP2) positive patients have a severe long-term prognosis but anti-Cll positive patients have transient inflammation. In the present study we wanted to extend this in a large RA cohort with clinical follow-up data, and to relate to HLA-DRB1* alleles.

Methods: Anti-CII and anti-CCP2 were measured at baseline in 773 patients from the Swedish Epidemiological Investigations in Rheumatoid Arthritis (EIRA) study with clinical follow-up data from the Swedish Rheumatology Quality (SRQ) registry, and 1476 patients with HLA-DRB1* information. Comparisons were done concerning CRP, ESR, TJC, SJC, DAS28, DAS28CRP, pain-VAS, global-VAS and $\mathrm{HAQ}$ at 8 occasions during 5 years, and association to HLA-DRB1* alleles.

Results: Anti-Cll was detected in 6.6\% (97/1476), and anti-CCP2 in 57.9\% $(855 / 1476)$ of the patients. There was no significant difference in treatment strategy at diagnosis for patients with and without those antibodies. Anti-Cll associated with elevated CRP, ESR, SJC, DAS28 and DAS28CRP at diagnosis and up to six months, whereas anti-CCP2 associated with SJC and DAS28 from 6 months to 5 years, but not earlier. The anti-Cll-associated phenotype was strong, and predominated also in anti-CII/anti-CCP2 double positive patients. Compared to baseline levels, anti-CII was asscociated with improvements in CRP, ESR, SJC, TJC and DAS28 over time, whereas anti-CCP2 was associated with deteriorations in SJC and DAS28 over time, compared to antibody negative patients. Anti-CII positive patients achieved EULAR good or moderate response more often than negative patients whereas the opposite was found for anti-CCP2 positive patients (figure). Anti-Cll was positively associated with HLADRB $1{ }^{*} 01$ and HLADRB $1{ }^{*} 03$, with significant interaction, and double positive individuals had $>14$ times higher mean anti-CII levels than HLA double negatives. Whereas smoking associated with elevated anti-CCP2 levels, smokers has lower anti-CII levels.

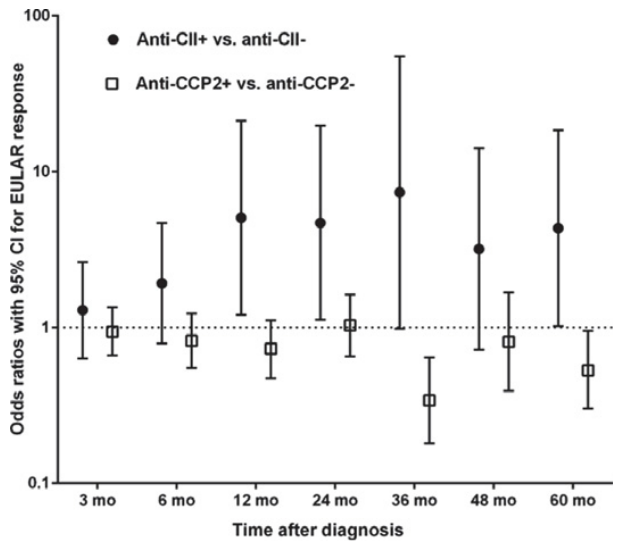

Conclusions: Anti-Cll seropositive RA represents a distinct phenotype, in many respects representing the converse to the clinical, genetic and smoking associations described for ACPA. Although not diagnostically useful, early anti-CII determinations predict favourable inflammatory outcome in RA. The combined analysis of anti-CII and ACPA/anti-CCP2 may be a new two-dimensional tool for predicting the prognosis and chosing therapy in newly diagnosed RA patients.

Disclosure of Interest: None declared

DOI: 10.1136/annrheumdis-2017-eular.2776

\section{THU0078 CONCOMITANT USE OF CONVENTIONAL SYNTHETIC DMARDS AND RESPONSE TO BARICITINIB}

A. Kavanaugh ${ }^{1}$, C. Helt ${ }^{2}$, D. Muram ${ }^{2}$, J. Alam ${ }^{2}$, V. Arora ${ }^{2}$, A.L. Pinto Correia ${ }^{2}$, I. de la Torre ${ }^{2}$, R. van Vollenhoven ${ }^{3}$. ${ }^{1}$ UC San Diego School of Medicine, la Jolla; ${ }^{2}$ Eli Lilly and Company, Indianapolis, United States; ${ }^{3}$ Amsterdam Rheumatology \& Immunol Center, Amsterdam, Netherlands

Background: Baricitinib (BARI), an oral JAK1/JAK2 inhibitor, is in development for patients (pts) with moderate to severe rheumatoid arthritis (RA)..$^{1,2}$

Objectives: This post-hoc analysis of two phase 3 studies assessed whether concomitant use of conventional synthetic disease-modifying antirheumatic drugs (csDMARDs) altered the response or safety outcomes to BARI in RA pts and evaluated the effect of concomitant corticosteroid use on the efficacy of BARI.

Methods: Pts with $\geq 6$ swollen and tender joints and no prior biologic DMARD use were enrolled. In RA-BEAM (NCT01710358), methotrexate (MTX)-inadequate responder (IR) pts were randomised to PBO once daily (QD), BARI $4 \mathrm{mg} Q D$, or adalimumab $40 \mathrm{mg}$ biweekly. ${ }^{1}$ In RA-BUILD (NCT01710358), csDMARD-IR pts were randomised to placebo (PBO) or BARI (2 or $4 \mathrm{mg}) \mathrm{QD}^{2}$ Pts continued background csDMARD (including MTX) therapy. This post-hoc analysis included the PBO $(\mathrm{N}=716)$ and BARI $4 \mathrm{mg}(\mathrm{N}=714)$ pts and assessed the number and type of concomitant csDMARDS and concurrent corticosteroid use.

Results: $71 \%, 21 \%$, and $6 \%$ of PBO pts were taking MTX alone, MTX + $\geq 1$ other csDMARD, and non-MTX csDMARDs, respectively; in BARI $4 \mathrm{mg}$ pts, the

Abstract THU0078 - Table 1. Efficacy and Safety Through 12 Weeks Based on Concomitant csDMARD Usage

\begin{tabular}{|c|c|c|c|c|c|c|}
\hline & \multicolumn{3}{|c|}{ Placebo $(\mathrm{N}=716)$} & \multicolumn{3}{|c|}{ Baricitinib $4 \mathrm{mg}(\mathrm{N}=714)$} \\
\hline & $\begin{array}{c}\text { MTX Alone } \\
(\mathrm{n}=506)\end{array}$ & $\begin{array}{c}\text { MTX }+\underset{(n=147)}{\geq 1 \text { csDMARDs }} \\
(n=140\end{array}$ & $\begin{array}{c}\text { Non-MTX csDMARDs } \\
(n=43)\end{array}$ & $\begin{array}{l}\text { MTX Alone }(n=527) \\
(n=527)\end{array}$ & $\begin{array}{c}\text { MTX }+\underset{(n=131)}{\geq 1 \text { csDMARDs }} \\
(n=1\end{array}$ & $\begin{array}{c}\text { Non-MTX csDMARDs } \\
(\mathrm{n}=42)\end{array}$ \\
\hline \multicolumn{7}{|l|}{ Efficacy measures } \\
\hline ACR20 & $210(42)$ & $57(39)$ & $17(40)$ & $361(69)$ & $85(65)$ & $25(60)$ \\
\hline ACR50 & $84(17)$ & $18(12)$ & $7(16)$ & $227(43)$ & $52(40)$ & $13(31)$ \\
\hline ACR70 & $20(4)$ & $7(5)$ & $3(7)$ & $106(20)$ & $19(15)$ & $5(12)$ \\
\hline $\mathrm{SDAl} \leq 11$ & $92(18)$ & $18(12)$ & $10(23)$ & $221(42)$ & $45(34)$ & $14(33)$ \\
\hline SDAI $\leq 3.3$ & $8(2)$ & $3(2)$ & 0 & $52(10)$ & $4(3)$ & $2(5)$ \\
\hline DAS28-ESR $\leq 3.2$ & $11(2)$ & $3(2)$ & $1(2)$ & $58(11)$ & $11(8)$ & $2(5)$ \\
\hline DAS28-ESR $<2.6$ & $35(7)$ & $10(7)$ & $4(9)$ & $129(24)$ & $27(21)$ & $7(17)$ \\
\hline \multicolumn{7}{|l|}{ Safety measures } \\
\hline$\geq 1$ adverse event & $442(87)$ & $132(90)$ & 39 (91) & $465(88)$ & $116(89)$ & $40(95)$ \\
\hline$\geq 1$ serious adverse event & $14(3)$ & $4(3)$ & $3(7)$ & $14(3)$ & $1(1)$ & $1(2)$ \\
\hline Discontinuation due to adverse event & $16(3)$ & $2(1)$ & 0 & $11(2)$ & $5(4)$ & $3(7)$ \\
\hline Death & $1(0.2)$ & $1(0.7)$ & 0 & 0 & 0 & 0 \\
\hline
\end{tabular}

Data are $\mathrm{n}(\%)$ 